\title{
ELLIPTICAL POLARIZATION BY LIGHT SCATTERING BY SUBMICRON SPHEROIDS
}

\author{
TH. SCHMIDT
}

Max-Planck-Institut für Astronomie, Heidelberg, Germany

\begin{abstract}
Calculations have been carried out of single light scattering by Rayleigh type spheroidal dust particles. It can be shown that elliptical polarized radiation with $e=v / p \approx 0.15$ can be produced from unpolarized incident radiation, in the case of dirty quartz particles with a refractive index $m=1.54 \pm 0.4 i$ and a geometrical axial ratio $c: a \approx 1: 2$.
\end{abstract}

\section{Introduction}

An elliptical polarimetry of the 30 Doradus and $\eta$ Carinae nebulae, undertaken with the Heidelberg telescope at the Boyden Observatory (South Africa) in the blue spectral region, has shown a circular polarized component greater than 1.5 times the rms error of measurement in 3 cases:

$$
\begin{aligned}
& 30 \text { Dor: }(v / p)_{1}=0.15 \pm 0.05 ;(v / p)_{2}=0.08 \pm 0.05 \\
& \eta \text { Car: }(v / p)_{3}=0.09 \pm 0.045
\end{aligned}
$$

$v$ and $p$ being the circular and linear amount of polarization respectively (see Schmidt, 1971).

The question arises whether it may be possible to explain these ellipticities $e=v / p$ by dust particle scattering, even in the case of unpolarized incident radiation and single scattering (i.e. considering optically thin dust clouds). A direct interpretation of the amount of circular polarization $v=V / I$ does not seem meaningful because the intensity $I$ is confused by strong gaseous emissions in both nebulae.

\section{Theory}

Concerning these questions calculations are presented of scattering properties of ellipsoidal submicron (i.e. Rayleigh like) particles.

Let $\boldsymbol{P}$ be the vector of the 4 Stokes' parameters

$$
\boldsymbol{P}=\left(\begin{array}{l}
I \\
Q \\
U \\
V
\end{array}\right)
$$

and $F$ the transformation matrix for an arbitrary optical process

$$
\boldsymbol{P}=F \cdot \boldsymbol{P}_{0}
$$


after van de Hulst (1957, p. 43 f.). For unpolarized incident light $I_{0} \neq 0, Q_{0}=U_{0}=$ $V_{0}=0$. In this case from a circular polarization parameter $V \neq 0$ follows the condition $F_{41} \neq 0$, while the ellipticity $e=v / p$ is identical to

$$
e=F_{41} / \sqrt{F_{21}^{2}+F_{31}^{2}}-
$$

The transformation $F$ has to be computed for the light scattering process by ellipsoidal Rayleigh particles (i.e. in the blue spectral region for particle diameters $d<0.13 \mu \mathrm{m}$ ).

In Figure 1 the co-ordinate notation of an arbitrarily oriented spheroid is illustrated. The $X, Y, Z$-system describes the geometry of scattering, $Z$ pointing towards the observer and $Z, Y$ being the plane of scattering. The co-ordinate system 1, 2, 3 is connected with the particle geometry, 3 being the axis of symmetry and 1 the intersection between the $X, Y$ - and 1, 2-plane; $\vartheta$ is the nutation and $\psi$ the precession angle of the particle in the $X, Y, Z$-system.

From the equations of van de Hulst (1957, pp. 44 and 78 f.) follows

$$
F_{41} \propto \operatorname{Im}\left(\alpha_{3} \alpha_{1}^{*}\right) \sin \psi \sin \vartheta \sin \theta\{\cos \psi \sin \vartheta \sin \theta+\cos \vartheta \cos \theta\}
$$

$\theta$ being the angle of scattering; $\alpha_{j}$ is the polarizability tensor of the particle $\left(\alpha_{j}^{*}\right.$ is the conjugate complex):

$$
\alpha_{j} \propto\left(1 /\left(m_{j}^{2}-1\right)+L_{j}\right)^{-1}
$$

$m_{j}$ being the complex refractivity tensor and $L_{j}$ a particle shape tensor. Some cases of $L_{j}$ are tabulated by van de Hulst $\left(1957\right.$, p. 72); for spheres is $L_{1}=L_{2}=L_{3}=\frac{1}{3}$, for flat rotationally symmetric disks $L_{1}=1, L_{2}=L_{3}=0$ and for needles $L_{1}=0$, $L_{2}=L_{3}=0.5 . F_{21}$ and $F_{31}$ are more complicated functions of $\alpha_{j}, \vartheta, \psi$ and $\theta$ than $F_{41}$.

From Equation (1) and (2) it can be concluded that $F_{41} \neq 0$ is possible only if (1) $L_{j} \neq L_{k}$ or $m_{j} \neq m_{k}$ for any $j \neq k$, and

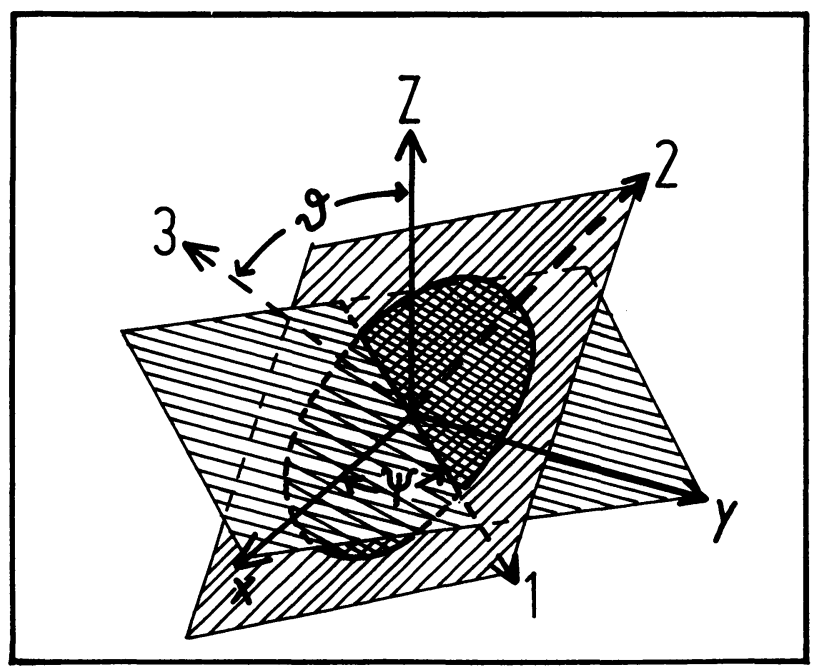

Fig. 1. Orientation of dust spheroids. $Z$ : direction to the observer, 3: particle axis of symmetry. 
(2) $m_{j}=n_{j}-\varkappa_{j} i$ with $a$ nonzero imaginary compenont $\varkappa_{j}$.

The maximum of $F_{41}$ occurs at $\vartheta=\theta=90^{\circ}, \psi=45^{\circ}$ and is equal to

$$
F_{41, \max } \propto \frac{1}{2} \operatorname{Im}\left(\alpha_{3} \alpha_{1}^{*}\right),
$$

but these angles would not produce generally the maximum of ellipticity

$$
F_{41} / \sqrt{F_{21}^{2}+F_{31}^{2}} \text { as well. }
$$

\section{General Results}

In the following calculations will be discussed in some detail for 'dirty quartz' type spheroids, i.e. a scalar refractive index

$$
m=1.54-x i \text {. }
$$

Figure 2 shows the computed ellipticities in case of $F_{41, \max }$ for different values of $\varkappa$ and axial ratios $c / a$ ( $c$ being the geometrical axis of symmetry). For values $c / a=1: 2$ or $2: 1 \varkappa \approx 0.4$ is necessary to explain the maximum observed ellipticity $e=0.15$ (somewhat smaller for the water ice case, shown for comparison).

In Figure 3 ellipticies are drawn for different angles $\theta, \vartheta$ and $\psi$, taking $m=1.54-$ $0.4 i$ and $c / a=0.5$ (i.e. oblate spheroids); some more detailed calculations of the same type are shown in Figure 4. It turns out that ellipticities $e \approx 0.5$ (and even of more than 1 ) are possible for angles, where $v$ itself has no maximum but the linear polarization is small.

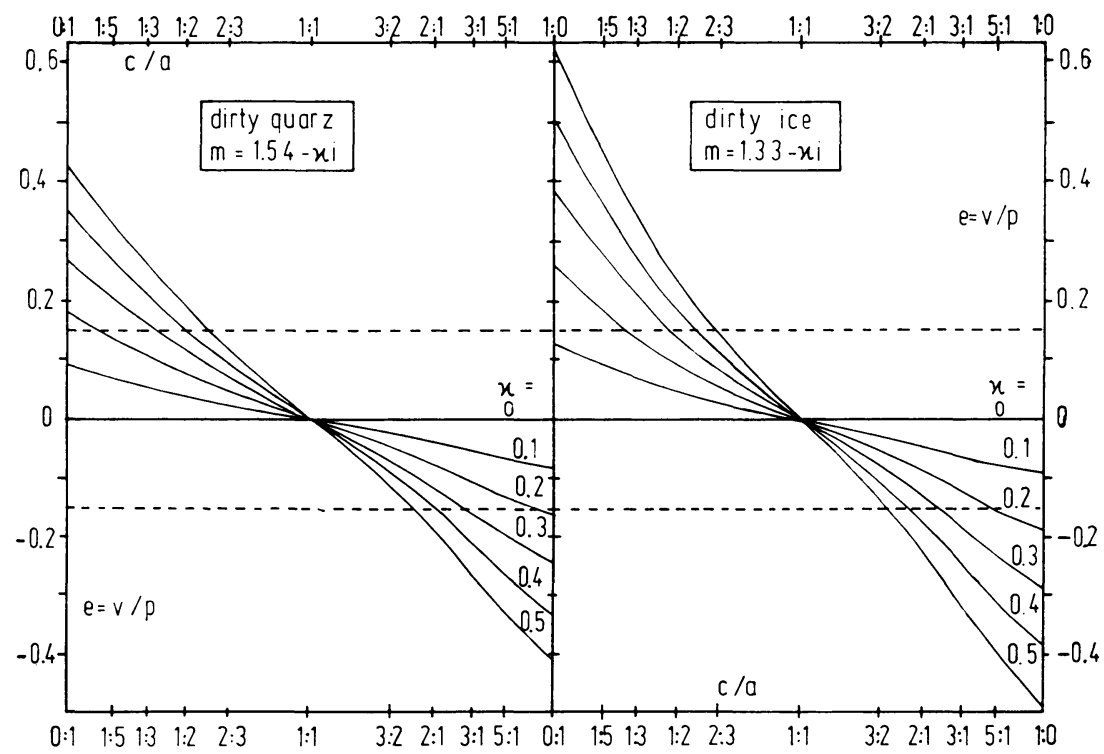

Fig. 2. Ellipticities $e=v / p=V / \sqrt{Q^{2}+U^{2}}$ by Rayleigh scattering at dirty quartz and ice spheroids in case of $V_{\max }=V\left(\psi=45^{\circ}, \vartheta=90^{\circ}, \theta=90^{\circ}\right)$ and different absorbing indices $\varkappa$ and axial ratios of the particle $c / a(c$ : axis of symmetry). 


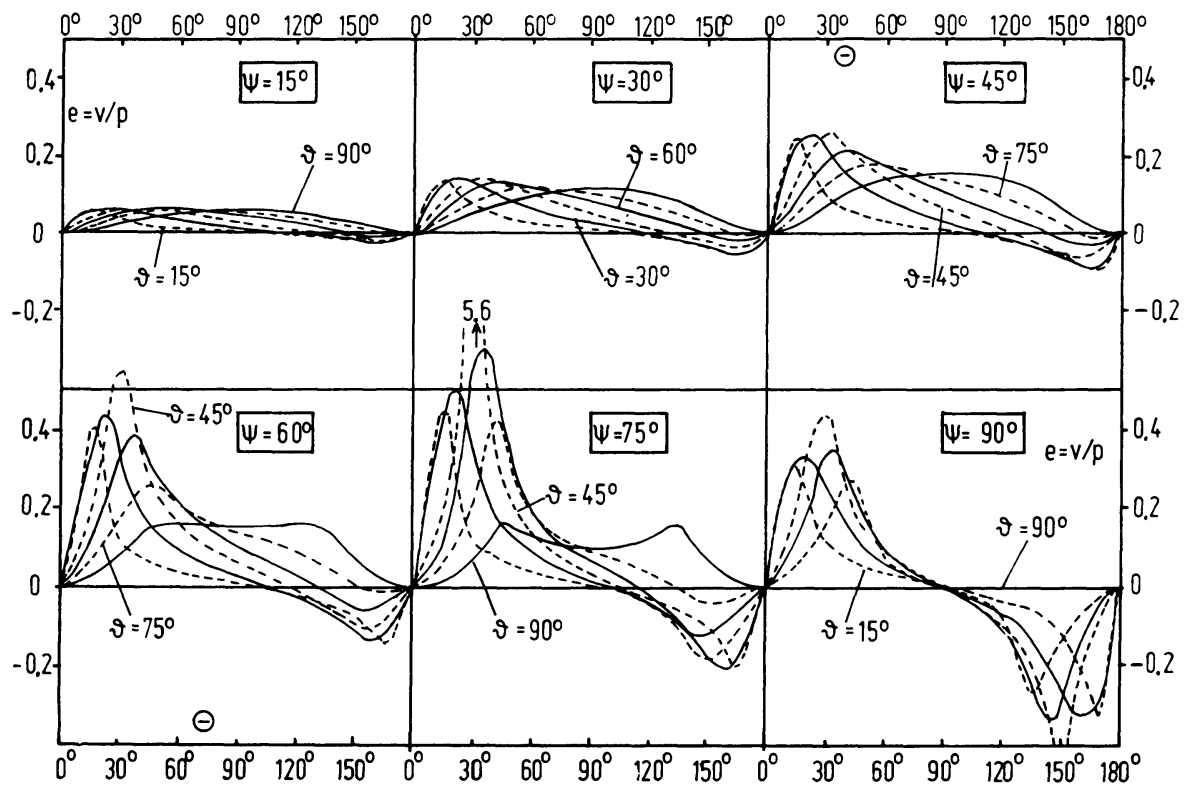

Fig. 3. Ellipticities $e=v / p$ by Rayleigh scattering at dirty quartz spheroids ( $m=1.54-0.4 i$ and $c / a=0.5$ ) for different angles of scattering $(\theta)$, nutation $(\vartheta)$ and precession $(\psi)$. (For angles $\psi^{\prime}=-\psi$ take: $e^{\prime}=-e$; for $\psi^{\prime}=180^{\circ}-\psi$ take: $e^{\prime}=-e$ and $\theta^{\prime}=180^{\circ}-\theta$; for $\vartheta^{\prime}=180^{\circ}-\vartheta$ take: $e^{\prime}=e$ and $\theta^{\prime}=180^{\circ}-\theta$.)

TABLE I

Polarization of scattered light from an optical thin spheric symmetrical cloud of dust particles corresponding to Figure 4. $\left(p=\sqrt{Q^{2}+U^{2}} / I ; v=V / I ; e=v / p ; \varphi\right.$ : polarization angle against tangential direction)

\begin{tabular}{|c|c|c|c|c|c|}
\hline \multirow{2}{*}{$\begin{array}{l}\vartheta \\
\psi\end{array}$} & \multicolumn{3}{|c|}{$80^{\circ}$} & \multicolumn{2}{|c|}{$90^{\circ}$} \\
\hline & & $45^{\circ}$ & $55^{\circ}$ & $45^{\circ}$ & $55^{\circ}$ \\
\hline \multirow{4}{*}{$r_{0}=0.5 r_{1}$} & $p$ & $58.7 \%$ & $52.3 \%$ & $59.1 \%$ & $52.5 \%$ \\
\hline & $\varphi$ & $-13^{\circ} .8$ & $-14^{\circ} .9$ & $-14^{\circ} .3$ & $-15^{\circ} .5$ \\
\hline & $v$ & $7.8 \%$ & $7.7 \%$ & $8.1 \%$ & $8.0 \%$ \\
\hline & $e$ & 0.133 & 0.148 & 0.137 & 0.153 \\
\hline \multirow{4}{*}{$r_{0}=r_{1}$} & $p$ & $71.2 \%$ & $66.3 \%$ & $71.6 \%$ & $66.4 \%$ \\
\hline & $\varphi$ & $-11^{\circ} .2$ & $-11^{\circ} .6$ & $-11^{\circ} .6$ & $-12^{\circ} .1$ \\
\hline & $v$ & $9.9 \%$ & $9.9 \%$ & $10.3 \%$ & $10.3 \%$ \\
\hline & $e$ & 0.138 & 0.149 & 0.143 & 0.156 \\
\hline \multirow{4}{*}{$r_{0}=2 r_{1}$} & $p$ & $84.9 \%$ & $82.2 \%$ & $85.3 \%$ & $82.3 \%$ \\
\hline & $\varphi$ & $-9^{\circ} .3$ & $-9^{\circ} .3$ & $-9^{\circ} .7$ & -9.7 \\
\hline & $v$ & $12.1 \%$ & $12.3 \%$ & $12.5 \%$ & $12.8 \%$ \\
\hline & $e$ & 0.142 & 0.150 & 0.147 & 0.156 \\
\hline \multirow{4}{*}{$\theta=90^{\circ}$} & $p$ & $98.6 \%$ & $98.6 \%$ & $98.9 \%$ & $98.8 \%$ \\
\hline & $\varphi$ & $-7^{\circ} .9$ & $-7^{\circ} .7$ & $-8^{\circ} .3$ & $-8^{\circ} .1$ \\
\hline & $v$ & $14.2 \%$ & $14.7 \%$ & $14.8 \%$ & $15.4 \%$ \\
\hline & $e$ & 0.144 & 0.149 & 0.149 & 0.156 \\
\hline
\end{tabular}




\section{A Spherically Symmetrical Dust Cloud}

The data of Figure 4 have been taken for calculating the properties of integrated polarisation of light scattered by a spheric symmetrical, optical thin dust cloud with a central illuminating star. In this case the integral

$$
\bar{P}(\vartheta, \psi) \propto \int_{-\infty}^{+\infty} P(\vartheta, \psi, \theta(Z)) \varrho(r) \frac{\mathrm{d} Z}{r^{2}}
$$

has to be calculated, $Z$ being the co-ordinate along the light beam, $\theta$ the angle of scattering and $r$ the distance from the central star; $\varrho$ is the dust density and $P$ represents the Stokes' parameters $I, Q, U, V$ or $F_{11}, F_{21}, F_{31}, F_{41}$ of the transformation matrix $F$ respectively. Assuming a Gaussian density distribution

$$
\varrho(r)=\varrho_{0} \exp \left(-r^{2} / r_{1}^{2}\right)
$$

and remembering that

$$
\tan \theta=r_{0} / Z, \quad \mathrm{~d} Z / \mathrm{d} \theta=-r_{0} / \sin ^{2} \theta \text { and } \sin \theta=r_{0} / r
$$

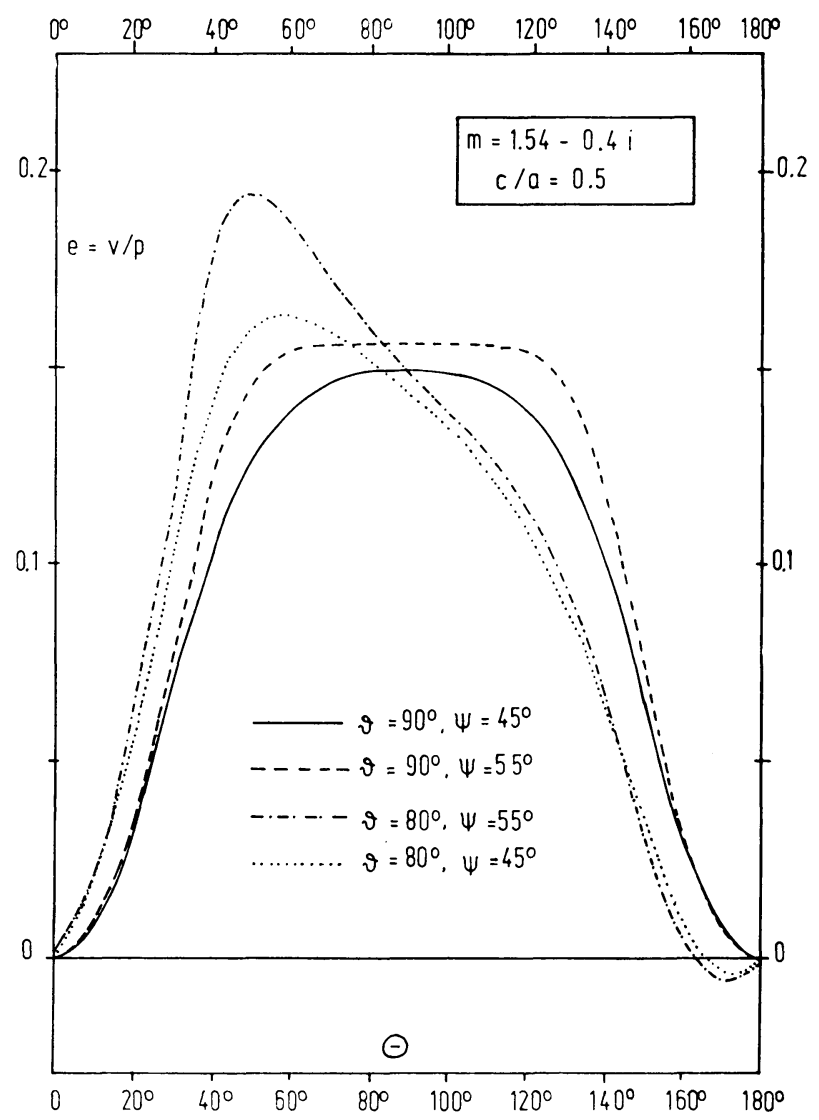

Fig. 4. Ellipticities $e=v / p$ for 4 special particle orientations (notations as in Figure 3). 
( $r_{0}$ being the minimum distance between light beam and central star), then

$$
\bar{P}(\vartheta, \psi) \propto \int_{0}^{\pi} P(\vartheta, \psi, \theta) \exp \left\{-\left(r_{0} / r_{1}\right)^{2} / \sin ^{2} \theta\right\} \mathrm{d} \theta .
$$

Table I shows the results for all cases drawn in Figure 4, together with the non integrated values of $\theta\left(v_{\max }\right)=90^{\circ}$ for comparison. Integrated ellipticities up to nearly $e=0.16$ ocour, while the deviation of polarization angles from tangential directions remains rather small.

\section{Spinning Particles}

According to the alignment by the mechanism of Davis and Greenstein (1951) the dust particles spin around their main axis of inertia. Therefore some supplementary test calculations have been carried out for a sample of prolate spheroids spinning around one of their small axes of geometry. As shown by Table II particles of that kind are between $35 \%$ and $50 \%$ less effective in producing circular polarization than oblate spheroids (the ratio of particle axes being $1: 2$ both).

Moreover it should be mentioned that a further reduction of ellipticity would occur, if (in case of weak magnetic fields, e.g.) the dust particles are not completely aligned.

\section{Conclusion}

It turns out that it is possible to produce elliptical polarized light up to $e=v / p \approx 0.15$ by single Rayleigh scattering processes, assuming aligned dirty quartz spheroids with a geometrical axial ratio of $1: 2$ and an absorbing part of the refractive index $\varkappa=0.4$ to 0.5 .

This value seems to be greater than suggested by the high interstellar dust albedo found from diffuse galactic light measurements by Witt (1968), Mattila $(1970,1971)$ and others. After Krishna Swamy et al. (1971) the albedo limited absorption of interstellar dust would be $\varkappa \approx 0.2$. On the other hand it seems to be not impossible that the dust medium connected with hot $\mathrm{H}$ II regions (like 30 Dor and $\eta$ Car) shows some

TABLE II

Ellipticity reduction factor $e_{p} / e_{0}$ for replacing oblate by spinning prolate spheroids (axial ratio $=1: 2$ both)

\begin{tabular}{|c|c|c|c|c|c|}
\hline \multirow[t]{2}{*}{$\vartheta$} & \multicolumn{5}{|c|}{$\psi, 180^{\circ}-\psi$} \\
\hline & $15^{\circ}$ & $30^{\circ}$ & $45^{\circ}$ & $60^{\circ}$ & $75^{\circ}$ \\
\hline $15^{\circ}$ & 0.66 & 0.65 & 0.64 & 0.64 & 0.63 \\
\hline $30^{\circ}$ & 0.64 & 0.63 & 0.62 & 0.61 & 0.59 \\
\hline $45^{\circ}$ & 0.63 & 0.62 & 0.60 & 0.58 & 0.56 \\
\hline $60^{\circ}$ & 0.63 & 0.60 & 0.58 & 0.55 & 0.53 \\
\hline $75^{\circ}$ & 0.62 & 0.60 & 0.56 & 0.53 & 0.50 \\
\hline $90^{\circ}$ & 0.62 & 0.60 & 0.56 & 0.52 & 0.49 \\
\hline
\end{tabular}


pecularities or that the actual ellipticity in 30 Dor is somewhat less than 0.15 , due to the rms error of \pm 0.5 .

Another open question is, how the situation turns out in case of Mie particles, although it seems not improbable that the general situation will not be more disadvantageous, especially as the elliptical polarization is due to individual particle properties being normally more marked in the Mie than in the Rayleigh particle region.

\section{Acknowledgements}

The calculations have been undertaken by the computer Siemens 2002 of the Heidelberg University.

I am indebted to Drs R. D. Wolstencroft and L. Bandermann for a valuable discussion which gave me occasion to find an error in calculating the linear polarization of the scattered radiation.

\section{References}

Davis, L. and Greenstein, J. L.: 1951, Astrophys. J. 114, 206.

Krishna Swamy, K. S., Jackson, W. M., and Donn, B. D.: 1971, Astron. Nachr. $293,43$.

Mattila, K.: 1970, Astron. Astrophys. 9, 53.

Mattila, K.: 1971, Astron. Astrophys. 15, 292.

Schmidt, Th.: 1971, Astron. Astrophys. 12, 456.

Van de Hulst, H. C.: 1957, Light Scattering by Small Particles, New York, London.

Witt, A. N.: 1968, Astrophys. J. 152, 59. 